\title{
Efeito da atividade física na qualidade do sono e qualidade de vida: revisão sistematizada
} Effect of physical activity on sleep quality and quality of life:

a systematized review

Efecto de la actividad física sobre la calidad del sueño y la calidad de vida: una revisión sistematizada

Lucilene Maria ROPKE ${ }^{1}$

Amanda Gouvea SOUZA²

André Pinheiro de Magalhães BERTOZ ${ }^{3}$

Manuel Martin ADRIAZOLA

Erika Verusca Paiva ORTOLAN ${ }^{5}$

Regina Helena MARTINS ${ }^{6}$

Weder Carneiro LOPES ${ }^{7}$

Cesar Diogo Benichio RODRIGUES ${ }^{8}$

Renato BIGLIAZZI ${ }^{3}$

Silke Anna Theresa WEBER ${ }^{6}$

${ }^{I}$ Fisioterapeuta, Mestranda Programa de Pós-Graduação Bases Gerais da Cirurgia, Faculdade de Medicina de Botucatu Univ Estadual Paulista - UNESP, 18618-687 Botucatu - SP, Brasil

${ }^{2}$ Graduada em Fisioterapia pelas Faculdades Adamantinenses Integradas (FAI)

${ }^{3}$ Professor Assistente Doutor, Departamento de Odontologia Infantil e Social, Faculdade de Odontologia de Araçatuba Univ Estadual Paulista - UNESP, 16015-050 Araçatuba - SP, Brasil

${ }^{4}$ Mestrando, Programa de Pós-Graduação em Odontologia, Área de Concentração Ortodontia,

Faculdade de Odontologia de Araçatuba, Univ Estadual Paulista - UNESP, 16015-050 Araçatuba - SP, Brasil

${ }^{5}$ Professora Adjunto, Departamento de Cirurgia e Ortopedia, Faculdade de Medicina de Botucatu Univ Estadual Paulista - UNESP, 18618-687 Botucatu - SP, Brasil

${ }^{6}$ Professora Adjunto, Departamento de Oftalmologia e Otorrinolaringologia, Faculdade de Medicina de Botucatu Univ Estadual Paulista - UNESP, 18618-687 Botucatu - SP, Brasil

${ }^{7}$ Cirurgião-Dentista pela Univ Estadual Paulista-UNESP, Mestrando em Implantodontia, Faculdade São Leopoldo Mandic, 13045-755 Campinas-SP, Brasil

${ }^{8}$ Graduando em Odontologia, Faculdade de Odontologia de Araçatuba Univ Estadual Paulista - UNESP, 16015-050 Araçatuba - SP, Brasil

\section{Resumo}

Introdução: O sono é um importante indicador de saúde e sua qualidade pode sofrer influência de diversos fatores, como alterações psicológicas e sociais; ambiente; uso de medicamentos; ingestão alcoólica e a prática regular de atividade física. Porém, ainda não está esclarecido se a atividade física teria efeito positivo sobre os distúrbios de sono mais prevalentes como insônia e apneia do sono. Objetivo: buscar publicações que falam da interação sono e atividade física, e estudos que esclareçam os efeitos da atividade física sobre a qualidade do sono e qualidade de vida. Material e Método: foi feito uma busca na literatura nas bases de dados: Biblioteca Virtual em Saúde (BVS), Scielo, Pubmed e Scopus usando os descritores Exercício; Sono; Qualidade de Vida; Apneia Obstrutiva do Sono. Resultados: foram discutidos sete artigos que abordam o assunto a atividade física como coadjuvante para a qualidade do sono mostrando que atividade física tem ação benéfica. Conclusão: a atividade física melhora a percepção subjetiva e objetiva da qualidade do sono e qualidade de vida, podendo ser utilizada como tratamento terapêutico nos tratamentos dos distúrbios do sono. Descritores: Exercício; Sono; Qualidade de Vida; Apneia Obstrutiva do Sono.

\section{Abstract}

Introduction: sleep is an important indicator for health, its quality may suffer influences of several factors as psychological and social disturbances, alcohol abuse, environment, drug intake, and, positively, physical activity. However, it is not clear if physical activity would have a positive effect for the most prevalent sleep disorders as insomnia, and sleep apnea. Objective: the aim of this study was to perform a review on the literature which discussed the interaction between sleep and physical activity, regarding the repercussion on sleep quality and quality of life. Material an method: the search strategy included the databases Biblioteca Virtual em Saúde (BVS), Scielo, PubMed and Scopus, using the descriptors: Exercise; Sleep; Quality of Life; Sleep Apnea, Obstructive. Results: seven articles met the inclusion criteria, discussing physical activity as a co-adjuvant treatment, all showing improvement of sleep quality and positive effects on quality of life. Conclusion: physical activity improves subjective perception and objective evaluations of sleep quality and quality of life, and should be considered as a co-adjuvant treatment for sleep disorders.

Descriptors: Exercise; Sleep; Quality of Life; Sleep Apnea, Obstructive.

\section{Resumen}

Introducción: El sueño es un importante indicador de salud y su calidad puede sufrir influencia de diversos factores, como alteraciones psicológicas y sociales; (en inglés). uso de medicamentos; la ingestión alcohólica y la práctica regular de actividad física. Sin embargo, aún no se ha aclarado si la actividad física tendría un efecto positivo sobre los trastornos del sueño más prevalentes como el insomnio y la apnea del sueño. Objetivo: buscar publicaciones que hablan de la interacción sueño y actividad física, y estudios que aclaren los efectos de la actividad física sobre la calidad del sueño y calidad de vida. Material y Método: se hizo una búsqueda en la literatura en las bases de datos: Biblioteca Virtual en Salud (BVS), Scielo, Pubmed y Scopus utilizando los descriptores Ejercicio; Sueño; Calidad de Vida; Apnea Obstructiva del Sueño. Resultados: fueron discutidos 7 artículos que abordan el asunto la actividad física como coadyuvante para la calidad del sueño mostrando que actividad física tiene acción benéfica. Conclusión: La actividad física mejora la percepción subjetiva y objetiva de la calidad del sueño y la calidad de vida, pudiendo ser utilizada como tratamiento terapéutico en los tratamientos de los disturbios del sueño.

Descriptores: Ejercicio; Sueño; Calidad de Vida; Apnea Obstructiva del Sueño.

\section{INTRODUÇÃO}

$\mathrm{O}$ bem estar, quanto às definições mais utilizadas atualmente pode ser dividido em duas formas: uma que aborda o estado subjetivo de felicidade conhecida como bem estar subjetivo, enquanto que a outra, trata-se do bem estar psicológico. Alguns autores consideram o bem estar subjetivo como uma avaliação da qualidade de vida ${ }^{1}$. A qualidade de vida é definida pela Organização Mundial da Saúde (OMS $)^{2}$ como "[...] a percepção do indivíduo de sua posição na vida no contexto da cultura e sistema de valores nos quais ele vive e em relação aos seus objetivos, expectativas, padrões e preocupações". A qualidade de vida reflete a subjetividade do resultado de um processo de 
construção no ambiente onde o indivíduo está inserido, tanto no contexto cultural como no social e não abrange somente fatores relacionados à saúde, inclui os pontos tanto positivos como negativos, deixando implícita a idéia do conceito multidimensional ${ }^{2,3}$.

O sono é considerado um processo biológico complexo mediado por modulações neural e hormonal, no qual alterna períodos de vigília atuando como restaurador das funções orgânicas, levando a uma condição essencial para o crescimento (infância e adolescência), e a aprendizagem / memória e funcionamento do organismo ${ }^{4}$. O sono relaciona-se diretamente com o estado de saúde e consequentemente com a qualidade de vida ${ }^{5}$. A percepção de qualidade de sono é descrita como dificuldade de dormir e de manter o sono; acordar muito cedo pela manhã; quanto renovada a pessoa se sente depois do sono; e a própria percepção do indivíduo sobre sua qualidade de sono. Alterações psicológicas, sociais, ambientais e fatores tais como: o uso de medicamentos; hábito de fumar; ingerir bebidas alcoólicas; e bebidas contendo cafeína com frequência, podem diminuir a qualidade de sono ${ }^{6}$.

Dentre os principais distúrbios do sono definidos na literatura encontram-se a insônia que é definida pelo Consenso Brasileiro de Insônia como "dificuldade de iniciar e/ou manter o sono e o sono não reparador, comprometendo as atividades diárias". Quando síndrome a insônia é considerada uma desordem primária que requer tratamento direto, como sintoma é secundária às condições médicas, psiquiátricas ou ambientais ${ }^{7}$. O segundo distúrbio mais frequentemente encontrado é a síndrome da apneia obstrutiva do sono(SAOS), uma síndrome caracterizada por paradas respiratórias totais (apneias) ou parciais (hipopneias) recorrentes de obstruções das vias aéreas superiores durante o sono, ronco excessivo, e despertares que fragmentam o sono favorecendo a ocorrência da hipersonolência diurna, cefaléia matinal, alterações neurocognitivas e piora na qualidade de vida. A apneia obstrutiva do sono está associada a diversas comorbidades como doenças cardiovasculares, diabetes, síndrome metabólica e apresenta fatores predisponentes comuns como a obesidade, o sexo masculino e a idade a partir dos 40 anos. Após suspeitar de SAOS com base na investigação dos sinais e sintomas é realizado o exame de polissonografia para estabelecer o diagnóstico ${ }^{8}$.

Estudos têm demonstrado que o exercício físico pode influenciar positivamente na qualidade de vida tanto em indivíduos com alguma patologia, quanto em populações saudáveis. A prática da atividade física pode influenciar o sono, repercutindo em aumento das ondas lentas e redução do sono REM. Além disso, a prática regular de exercícios físicos é uma intervenção não farmacológica recomendada pela Associação Americana de Sono para promover um sono adequado 9 . O propósito do presente trabalho é avaliar a correlação entre distúrbios do sono, atividade física e qualidade do sono por meio da busca de artigos na literatura.

\section{MATERIAL E MÉTODO}

A pesquisa foi realizada através da busca de artigos nas bases de dados da BVS (Lilacs e Medline), Pubmed, Scielo e Scopus. Os descritores utilizados para busca foram: exercício, qualidade de vida, sono e apneia obstrutiva do sono. Filtraram-se os artigos encontrados por duplicação nas bases de dados pelo título, resumo, idiomas (português, inglês, espanhol e alemão), se abordava o enfoque, e por último a análise na íntegra dos artigos considerados de interesse. Foram incluídos na pesquisa sete artigos atuais e mais relevantes que apresentam dados e resultados coerentes abordando o assunto, atividade física como coadjuvante no tratamento para a qualidade do sono. Para a inclusão o artigo tinha que conter relato de intervenção ou investigação sobre atividade física, relacionado-a com a avaliação da qualidade do sono em adultos, através de instrumentos validados (polissonografia, questionáriose escalas).

\section{RESULTADOS}

$\mathrm{Na}$ busca foram identificados 506 artigos, sendo 12 na base de dados BVS (Lilacs e Medline), 7 na base de dados Pubmed, 11 na base de dados Scielo e 476 na base de dados Scopus. Sendo excluídos os que não abordavam o assunto em questão (atividade física, qualidade do sono e adultos). Ao todo, os resumos de 248 artigos foram lidos e 22 artigos foram incluídos para a leitura na íntegra, destes 7 artigos foram selecionados, levando em consideração os instrumentos de avaliação da qualidade de sono e qualidade de vida, a população e o tipo de atividade realizados nos respectivos estudos. O objetivo dos estudos mencionados, instrumentos de avaliação, intervenção realizada e conclusão são apresentados na Tabela 1 .

Tabela 1. Relação dos artigos selecionados quanto aos autores, objetivo, avaliação, intervenção e conclusão

\begin{tabular}{|c|c|c|c|c|}
\hline Autores & Objetivo & Avaliação & Intervenção & Conclusão \\
\hline $\begin{array}{l}\text { Barnes et } \\
\text { al. }^{10}\end{array}$ & $\begin{array}{l}\text { Avaliar um programa } \\
\text { de exercício físico } \\
\text { associado a dieta em } \\
\text { pacientes obesos com } \\
\text { SAOS. }\end{array}$ & $\begin{array}{l}\text { Polissonografia; } \\
\text { ESS, BDI, POMS, } \\
\text { FOSQ, SASQ e } \\
\text { SF- } 36 \text {. }\end{array}$ & $\begin{array}{l}\text { Exercício de } \\
\text { resistência, } \\
\text { exerć́cio } \\
\text { aeróbico e } \\
\text { dieta de baixa } \\
\text { caloria. }\end{array}$ & $\begin{array}{l}\text { Houve perda de } \\
\text { peso significativa, } \\
\text { redução nos } \\
\text { distúrbios } \\
\text { respiratórios do } \\
\text { sono e do IAH. }\end{array}$ \\
\hline Kline et al. ${ }^{11}$ & $\begin{array}{l}\text { Estudar a eficácia do } \\
\text { exercício na melhora } \\
\text { da severidade da } \\
\text { SAOS. }\end{array}$ & $\begin{array}{l}\text { Polissonografia; } \\
\text { BQ, PSQI, e } \\
\text { REAP-S. }\end{array}$ & $\begin{array}{l}\text { Exercício de } \\
\text { resistência, } \\
\text { exerćício } \\
\text { aeróbico e } \\
\text { alongamento. }\end{array}$ & $\begin{array}{l}\text { O treinamento físico } \\
\text { auxiliou na redução } \\
\text { do IAH e pode ser } \\
\text { benéfico por facilitar } \\
\text { a perda de peso. }\end{array}$ \\
\hline Kline et al. ${ }^{12}$ & $\begin{array}{l}\text { Explorar a utilidade do } \\
\text { exercício físico na } \\
\text { melhora do } \\
\text { desempenho das } \\
\text { atividades diurnas em } \\
\text { adultos com SAOS. }\end{array}$ & $\begin{array}{l}\text { Polissonografia; } \\
\text { BQ, ESS, SF-36, } \\
\text { CES-D, } \\
\text { FOSQ,PVT, } \\
\text { SCTW, TMT, e } \\
\text { POMS. }\end{array}$ & $\begin{array}{l}\text { Exercício de } \\
\text { resistência, } \\
\text { exerćício } \\
\text { aeróbico e } \\
\text { alongamento. }\end{array}$ & $\begin{array}{l}\text { O exercício pode } \\
\text { melhorar aspectos } \\
\text { de desempenho } \\
\text { das atividades } \\
\text { diurnas nos adultos } \\
\text { com SAOS. }\end{array}$ \\
\hline 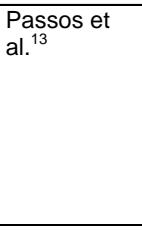 & $\begin{array}{l}\text { Estudar em uma } \\
\text { revisãa sistemática, se } \\
\text { o exercício físico é } \\
\text { uma alternativa de } \\
\text { tratamento para } \\
\text { insônia crônica. }\end{array}$ & $\begin{array}{l}\text { Artigos publicados } \\
\text { entre } 1983 \text { e } 2011 \\
\text { usando Medline, } \\
\text { Scopus e Web of } \\
\text { Science. }\end{array}$ & $\begin{array}{l}\text { Palavras } \\
\text { chave: } \\
\text { Insônia, Sono, } \\
\text { Queixas de } \\
\text { Sono, } \\
\text { Exercício e } \\
\text { Atividade } \\
\text { Física. }\end{array}$ & $\begin{array}{l}\text { A atividade física } \\
\text { mostrou-se eficaz } \\
\text { no tratamento de } \\
\text { insônia crônica. }\end{array}$ \\
\hline $\begin{array}{l}\text { Pereira et } \\
\text { al. }^{14}\end{array}$ & $\begin{array}{l}\text { Estimar a prevalência } \\
\text { da SDE e identificar os } \\
\text { fatores associados. }\end{array}$ & $\begin{array}{l}\text { ESS, BQ, BDI,BAI } \\
\text { e BKQ. }\end{array}$ & $\begin{array}{l}\text { Sem } \\
\text { intervenção. }\end{array}$ & $\begin{array}{l}\text { Atividade física } \\
\text { acima da média } \\
\text { pode significar } \\
\text { sobrecarga de } \\
\text { atividades, } \\
\text { favorecendo } \\
\text { aumento da SDE. }\end{array}$ \\
\hline $\begin{array}{l}\text { Asharafinia } \\
\text { et al. }{ }^{15}\end{array}$ & $\begin{array}{l}\text { Investigar os efeitos } \\
\text { de exercícios de } \\
\text { Pilates sobre a } \\
\text { qualidade do sono no } \\
\text { pós-parto de } \\
\text { primigestas. }\end{array}$ & PSQI & $\begin{array}{l}\text { Exercício de } \\
\text { pilates. }\end{array}$ & $\begin{array}{l}\text { Exercícios de } \\
\text { Pilates } \\
\text { proporcionaram } \\
\text { uma melhora na } \\
\text { qualidade do sono. }\end{array}$ \\
\hline $\begin{array}{l}\text { Monteiro et } \\
\text { al. }\end{array}$ & $\begin{array}{l}\text { Verificar a associação } \\
\text { entre sintomas de } \\
\text { insônia e cochilo } \\
\text { diurno e a participacaão } \\
\text { em atividades físicas } \\
\text { de lazer em idosos. }\end{array}$ & $\begin{array}{l}\text { MMSE, MLAQ e } \\
\text { questōes } \\
\text { extraídas do NHP. }\end{array}$ & $\begin{array}{l}\text { Investigar } \\
\text { atividade } \\
\text { física de lazer } \\
\text { prevalente. }\end{array}$ & $\begin{array}{l}\text { A caminhada pode } \\
\text { constituir-se de uma } \\
\text { intervenção que } \\
\text { favorece o padrão } \\
\text { de sono dos idosos. }\end{array}$ \\
\hline
\end{tabular}

Barnes et al. ${ }^{10}$ realizaram um estudo de viabilidade com um grupo de 21 pacientes obesos, adultos portadores de SAOS índice de apneia hipopneia (IAH) de 10 a 50, com predominância do sexo feminino, sem comorbidades significativas, e capazes de realizar exercício físico. $\mathrm{Na}$ avaliação inicial realizaram a polissonografia $(P S G)$, aplicaram os questionários: Epworth Sleepiness Scale (ESS), Beck Depression Index (BDI), Profile Of Mood States (POMS), Functional Outcomes of Sleep Questionnaire (FOSQ), Sleep Apnea Symptom Questionnaire (SASQ) e Medical Outcomes Study 36 - Item Short - Form Health Survey (SF-36). Todos os indivíduos fizeram o Cardiopulmonary exercise testing (CPET) e 
tiveram uma primeira entrevista com o nutricionista para discussão de sua atual dieta e um possível programa de dieta. O programa de exercício de 16 semanas incluía atividade aeróbica e treinamento de resistência, associados a uma dieta de muito baixa energia, sendo subdividido da seguinte maneira: $\mathrm{Da} 1^{\mathrm{a}}$ a $8^{\mathrm{a}}$ semana foram realizadas 3 sessões de exercícios supervisionados e de 2 a 4 sessões em casa. Após a $8^{\text {a }}$ semana, de 1 a 3 sessões foram supervisionadas e de 4 a 5 sessões em casa, e, a partir da $5^{\text {a }}$ sessão, foi incluído o treinamento aeróbico. $\mathrm{Da} 1^{\mathrm{a}}$ a $8^{\mathrm{a}}$ semana os indivíduos realizaram três refeições da dieta de baixa energia por dia, da $9^{\mathrm{a}}$ a $12^{\mathrm{a}}$ semana duas refeições por dia e da $13^{\mathrm{a}}$ semana em diante uma refeição por dia. $\mathrm{O}$ cálculo para a dieta baseou-se na taxa metabólica de repouso e o gasto energético estimado a partir do programa de exercício. $\mathrm{O}$ estudo visou avaliar distúrbios respiratórios do sono, o risco cardiovascular, e a função neurocomportamental. Evidenciou-se melhorias significativas nos resultados neurocomportamentais, cardiometabólicos e o ronco melhorou na maioria dos indivíduos. Os resultados deste estudo mostraram significativa perda de peso e melhora clínica importante, mudança nos distúrbios respiratórios do sono e o IAH foi inferior. $\mathrm{O}$ estudo concluiu que um programa de dieta $\mathrm{e}$ exercício físico pode ser um benefício para pacientes obesos com apneia do sono leve a moderada.

Kline et al. ${ }^{11}$ buscaram entender e explorar os possíveis mecanismos pelos quais o exercício pode reduzir a severidade da SAOS, avaliaram por 12 semanas a eficácia de um programa de exercício físico para melhorar o índice da apneia obstrutiva do sono e a melhora da qualidade do sono. Participaram do estudo 43 adultos, idade entre 18-55 anos, obesos sedentários com pelo menos SAOS moderada não tratada. Os participates realizaram Polissonografia $(P S G), \quad$ responderam aos questionários: Berlin Questionnaire (BQ), Pittsburgh Sleep Quality Index (PSQI), Rapid Eating Assessment for Participants-Short Version (REAP-S). Também foram avaliadas mudanças na composição corporal, função pulmonar e força muscular respiratória, por serem possíveis mediadores entre treinamento físico e melhoria do IAH. Foram escolhidos aleatoriamente 27 participantes para treinamento físico, sendo atividade aeróbica de intensidade moderada, seguido de treinamento de resistência. Estes realizaram um programa de exercícios supervisionados de 4 vezes por semana durante 12 semanas. Para limitar o risco de lesão a intensidade dos exercícios foi aumentada gradualmente durante as 4 semanas iniciais. Inicialmente de 50 minutos de esteira aumentando gradativamente chegando a 150 minutos na 5 semana e mantendo até a $12^{\mathrm{a}}$ semana. Para o grupo controle 16 participantes foram escolhidos aleatoriamente, estes realizaram apenas exercícios supervisionados de alongamento global para treinamento de flexibilidade, duas vezes por semana durante 12 semanas com duração de $60 \mathrm{~min}$. Quando comparado com o grupo controle, o grupo que realizou exercícios aeróbicos e de resistência apresenta uma significativa redução IAH, bem como alterações significativas no índice de dessaturação de oxigênio(IDO) e melhorias na macroestrutura do sono. As reduções no IAH e IDO foram alcançados sem uma diminuição significativa no peso corporal. Portanto concluiu-se que o treinamento físico repercutiu na redução do IAH em adultos obesos, o que sugere que o exercício pode ser benéfico para a gestão da SAOS além de simplesmente facilitar a perda de peso.

Kline et al. ${ }^{12}$ em segundo estudo com o mesmo grupo de indivíduos do trabalho anteriormente mencionado realizaram um estudo para explorar também a utilidade do exercício físico na melhora do desempenho das atividades diurnas em adultos com apneia obstrutiva do sono. Neste estudo foi avaliado o desempenho diurno e desempenho das atividades antes e após a intervenção com aplicação de questionários validados. Foi realizado polissonografia, aplicado os questionários: Profile of Mood States (POMS), Epidemiological Studies-Depression (CES-D), The Medical Outcomes Study 36-item- Short Form Health Survey (SF36), Epworth Sleepiness Scale (ESS), Functional Outcomes of Sleep Questionnaire (FOSQ-10), e realizadado os segunites testes: Psychomotor Vigilance Task (PVT), Stroop Cor-word Test(SCTW), Trail-Making Test(TMT) \} antes e após as intervenções. Comparando com o grupo controle que só realizou alongamento, o grupo que realizou o treinamento com exercício de resistência apresentou melhorias significativas nos sintomas depressivos e nos aspectos de qualidade de vida. Sonolência e prejuízo funcional melhoraram nos dois grupos, exercício versus controle, em um grau semelhante, em termos de tamanho e de efeito, embora estas alterações não foram estatisticamente significativas. Não houve melhora significativa da gravidade da SAOS nos dois grupos. O estudo concluiu que esses dados fornecem evidências preliminares de que o exercício físico pode ser útil para melhorar aspectos de atividades diurnas em adultos com SAOS. Entretanto, estudos maiores são necessários para comprovar os ganhos apresentados.

Passos et al. ${ }^{13}$ buscaram estudar em uma revisão sistemática, se o exercício físico é uma alternativa de tratamento para insônia crônica. Foram identificados estudos publicados entre 1983 e 2011 usando Medline, Scopus e Web of Science. Para a análise sistemática apenas os estudos que avaliam os efeitos crônicos do exercício sobre o sono em pessoas com queixas de sono ou pacientes com doenças crônicas como a insônia foram considerados. Utilizaram as seguintes palavras chaves: Insônia, Sono, Queixas de Sono, Exercício e Atividade Física. Identificaram 216 estudos inicialmente, mas apenas 5 preenchiam o critério de inclusão. Dois dos estudos investigaram idosos com queixas de sono e os outros 3 estudos investigavam pacientes com insônia primária crônica. O propósito da revisão sistemática era identificar os estudos sobre os efeitos da atividade física na melhora de queixas de insônia crônica em adultos de meia-idade e mais velhos, comparando os resultados com os obtidos no uso de medicamentos hipnóticos e ainda discutir possíveis mecanismos pelos quais a atividade física poderia promover o sono em pacientes com insônia. Verificaram que a atividade física mostrou-se um tratamento eficaz apresentando resultados semelhantes quando comparada com hipnóticos no tratamento de insônia crônica, sendo o exercício aeróbico o mais extensivamente estudado. Encontraram também evidências adicionais documentadas sobre os efeitos da atividade física como antidepressivos e anti-ansiedade.

Pereira et al. ${ }^{14}$ fizeram um estudo transversal com 372 mulheres com objetivo de estimar a prevalência da sonolência diurna excessiva (SDE) e identificar os fatores associados em mulheres de 35 a 49 anos de idade do Projeto de Saúde de Pindamonhangaba (PROSAPIN). A sonolência excessiva diurna, estilo de vida e comorbidades foram avaliados na entrevista utilizando instrumentos validados: Epworth Sleepiness Scale (ESS) para a sonolência excessiva diurna, Beck Depression Inventory (BDI) para depressão, 
Beck Anxiety Inventory (BAI) para ansiedade, Classificação da Obesidade pela Organização Mundial da Saúde para obesidade, Berlin Questionnaire (BQ) para a apneia obstrutiva do sono, Baecke Questionnaire (BKQ) para atividade física habitual, e Primeiro Levantamento Nacional sobre padrões de consumo de álcool e fumo na população brasileira para tabagismo e etilismo. Foram realizadas mensurações antropométricas (peso, altura, circunferências de quadril e abdômen), juntamente com entrevistas, para obter informações sobre o sono (latência e número de horas dormidas), as características sociodemográficas, a história ginecológica e o uso de medicamentos que podem alterar o estado de alerta. A prevalência da SDE foi de $18,5 \%$ e como fatores associados destacaram-se: a profissão relacionada a serviços domésticos, nível de atividade física acima da média da população estudada e a presença de características sugestivas de ansiedade. $\mathrm{O}$ estudo concluiu que a incidência da SDE nas mulheres é elevada nessa faixa etária e está associada a características sociodemográficas, à presença de comorbidades e ao estilo de vida. Quanto a relação entre a SDE e atividade física, concluíram que um nível de atividade física acima da média esperada para uma população específica pode significar sobrecarga de atividades, favorecendo aumento da SDE.

Ashrafinia et al. $^{15}$ realizaram um estudo com o objetivo de investigar os efeitos de um programa de treinamento de Pilates sobre a qualidade do sono em mulheres no pós-parto primigestas em um ensaio clínico randomizado. Participaram 80 mulheres que foram divididas aleatoriamente em grupos de intervenção e controle. Neste estudo, foram selecionados 13 exercícios de Pilates concentrando-se em respiração profunda, exercícios globais de movimentos de flexão-extensão, aquecimento e desaquecimento antes e após os exercícios. As participantes começaram os exercícios 72 horas até 1 semana após o parto e mantiveram durante 8 semanas. Os exercícios foram realizados no início da manhã por 30 minutos após aleitamento materno, cinco dias por semana (de preferência consecutivamente). A repetição e a intensidade dos exercícios foram gradualmente aumentada ao longo do programa. O grupo controle forneceu informações sobre qualquer atividade física além de suas atividades diárias de rotina para critérios de exclusão. As participantes selecionadas no grupo controle receberam folheto explicativo com o propósito de auto-aprendizagem e uma sessão de formação sobre cuidados pós-natais, a fim de manter a motivação de participação. Todas as participantes foram acompanhadas por telefonemas semanais para novas consultas. A qualidade do sono foi avaliada pelo Pittsburgh Sleep Quality Index (PSQI) antes e depois da intervenção. $O$ grupo de intervenção mostrou uma melhora significativa na qualidade subjetiva do sono, latência do sono, disfunção diurna e escore global do (PSQI), sugerindo que os exercícios de Pilates proporcionaram uma melhora na qualidade do sono em mulheres primigestas no pós-parto.

Monteiro et al. ${ }^{16}$ afirmam que a prática de atividade física contribui para redução do risco de doenças crônicas e melhora do padrão de sono em idosos. A pesquisa buscou verificar uma possível associação dos sintomas de insônia e cochilo diurno com a participação em atividade física de lazer. Foram extraídos dados da Rede de Estudos da Fragilidade em Idosos Brasileiros (FIBRA), do banco pertinente ao município de Campinas. Os participantes responderam um questionário de identificação e caracterização sociodemográfica construído pelos pesquisadores do estudo FIBRA, uma avaliação pelo mini mental state examination (MMSE) e questões sobre sintomas de insônia, apenas permaneciam no estudo os idosos que no (MMSE) indicavam ausência de déficit cognitivo. Neste estudo participaram 689 idosos e foram analisadas as variáveis seguintes: realização de atividades físicas de lazer, ocorrência de cochilo diurno e duração do cochilo diurno, questões extraídas do Minnesota Leisure Activity Questionnaire (MLAQ) validado para uso no Brasil; sintomas de insônia e uso de medicação para dormir, questões extraídas do Nottingham Health Profile (NHP), validado para uso no Brasil. Como resultados, observaram que a maioria dos participantes eram do sexo feminino, a idade em média 72 anos, quase metade dos idosos referiu pelo menos um sintoma de insônia, sendo mais prevalentes o despertar precoce e a dificuldade em iniciar o sono. $\mathrm{O}$ uso de medicamentos para dormir foi relatado por cerca de $20,0 \%$ das pessoas e a maior parte referiu cochilar durante o dia. Foi verificada a prevalência de cochilos com duração inferior a 90 minutos entre os idosos que praticavam a caminhada. A atividade física de lazer mais prevalente entre os idosos participantes deste estudo foi a caminhada e essa prática apresentou associação significativa com a duração do cochilo. Portanto, promover a pratica de caminhada pode constituir-se de uma intervenção que favorece o padrão de sono das pessoas idosas.

\section{DISCUSSÃO}

A pesquisa em questão surgiu da necessidade de conhecimento sobre os estudos realizados em adultos portadores de distúrbios do sono relacionados de alguma forma com atividade física, bem como o tipo de instrumentos padronizados utilizados para as avaliações.

Os distúrbios do sono tem aumentado sua prevalência na sociedade moderna, evidenciando-se o aumento da procura por parte dos pacientes de orientações médicas, devido à repercussão importante, sobre a qualidade de vida das pessoa acometidas. Entre os principais distúrbios cursam a insônia, seja pelo encurtamento do tempo de sono por ocupação, principalmente pelo uso constante dos meios eletrônicos, seja por diminuição da qualidade do sono, por estresse. Outro distúrbio muito prevalente é a síndrome da apneia obstrutiva do sono, a sua prevalência é proporcionada pelo aumento da obesidade.

Levando em consideração os estudos apresentados (Tabela 1), observa-se que os dados são isolados, não há um consenso que aborde todas as situações de distúrbios do sono tendo a atividade física como intervenção.

Nota-se que poucos estudos fizeram uma intervenção e controle de boa qualidade utilizando protocolos de estudos padronizados. Não há sobreposição do tipo populacional estudado, tipo de distúrbio do sono e do protocolo de avaliação de atividade proposta, ou seja, há uma dispersão de intervenções. Observa-se também que cada estudo realizou um tipo de intervenção, mostrando-a ser muito heterogênea e não permitindo uma somação ou uma comparação dos dados.

Os estudos não apresentam semelhança de população, entre eles estão mulheres pós parto, idosos, portadores de SAOS, portadores de insônia e mulheres de meia idade.

Em relação à avaliação da qualidade do sono, os instrumentos utilizados não foram os mesmos, verifica-se que os grupos utilizaram diferentes questionários para a avaliação subjetiva, até mesmo com a elaboração de um próprio protocolo (perguntas subjetivas), o que dificulta a 
compilação de dados, não sendo possível somar evidências. Ao todo, a intervenção da atividade física tem sido favorável quanto a percepção objetiva e subjetiva de qualidade do sono.

Nos estudos realizados por Passos et $\mathrm{al}^{13}$, Asharafinia et $\mathrm{al}^{15}$, Monteiro et $\mathrm{al}^{16}$ com pacientes portadores de insônia, mulheres pós parto e idosos, respectivamente as propostas da atividade física tiveram uma resolubilidade terapêutica apresentando resultados de melhora sem outra intervenção com medicamentos ou indutores do sono. No estudo de Pereira et $\mathrm{al}^{14} \mathrm{com}$ mulheres meia idade observa-se uma ressalva importante " a atividade física quando praticada em excesso pode favorecer o aumento da SDE ".

Para pacientes portadores de SAOS, a atividade física pode ser um tratamento adicional na melhora do IAH, da qualidade do sono e qualidade de vida, ou seja, há necessidade de reeducação de hábitos para que possam se beneficiar. Os estudos Barnes et $\mathrm{al}^{10}$, Kline et $\mathrm{al}^{11}$ e Kline et $\mathrm{al}^{12}$ que abordaram este problema e que realizaram a polissonografia pré e pós intervenção apresentaram redução do IAH para quem fez a atividade física (atividade aeróbica e alongamento), sendo ainda mais significativa quando a atividade física foi associada a uma dieta de baixa caloria, mas eles não permitem saber o quanto a intervenção foi eficaz. Esses estudos apresentaram como resultados objetivos dados polissonográficos como a diminuição significativa no índice de dessaturação de oxigênio, melhoria na fase N3(eficiência do sono) e como resultados subjetivos também apresentaram melhoras nos resultados dos questionários padronizados. Nenhum dos estudos relatou ter dado continuidade ao acompanhamento para mostrar se a intervenção foi duradoura no sentido de induzir a mudança de hábito de vida na população estudada.

É importante a realização de mais estudos voltados para a diminuição dos problemas relacionados aos distúrbios do sono e sobre os efeitos da atividade física na qualidade do sono, demonstrando a influência de fatores como a intensidade, a duração e o tipo de exercício, já que, além de atuar diretamente nas alterações fisiológicas benéficas, ela proporciona benefícios importantes no estado físico, social e psicológico dos seus praticantes.

\section{CONCLUSÃO}

Há evidências, embora ainda fracas, que a intervenção com atividade física melhore a percepção subjetiva e objetiva da qualidade do sono, podendo ser utilizada como tratamento terapêutico isolado ou como coadjuvante nos tratamentos dos distúrbios do sono. Faltam, porém, estudos delineados, randomizados e com a utilização de protocolos padronizados para fortalecer essas evidências.

\section{REFERÊNCIAS}

1. Albuquerque A S, Tróccoli B T. Desenvolvimento de Uma Escala de Bem-Estar Subjetivo. Psic: Teor e Pesq. 2004; 20(2):153-64

2. Esteves AM, Silva A, Barreto A, Cavagnolli DA, Ortega LSA, Parsons A et al. Avaliacao da qualidade de vida e do sono de atletas paralimpicos brasileiros. Rev Bras Med Esporte. 2015; 21(1):53-6.

3. Siqueira MMM, Padovam VAR. Bases Teóricas de Bem-Estar Subjetivo, Bem-Estar Psicológico e Bem-Estar no Trabalho. Psic: Teor e Pesq. 2008; 24(2):201-9.

4. Zanuto EAC, Lima MCS, Araújo RG, Silva EP, Anzolin CC, Araujo MYC et al. Distúrbios do sono em adultos de uma cidade do Estado de São Paulo. Rev Bras Epidemiol. 2015; 18(1):42-53.

5. Mediano O, Barceló A, de la Peña M, Gozal D, Agusti A, Barbé F. Hipersonolência diurna e variáveis polissonográficas em doentes com síndrome da apneia do sono. Rev Port Pneumol. 2007; 13(6):896-8.

6. Zanetti MC, Lavoura TNL, Machado AA. O treinamento desportivo e a qualidade de sono de atletas profissionais. http://www.efdeportes.com/ Revista Digital - Buenos Aires - Año 12 - $\mathrm{N}^{\circ} 110$ - Julio de 2007

7. Bacelar A, Pinto Jr LR. Insônia do diagnóstico ao tratamento. III Consenso Brasileiro de Insônia. São Paulo: Omnifarma Ltda; 2013.

8. Haddad F, Bittencourt L. Recomendações para o Diagnóstico e Tratamento da Síndrome da Apneia Obstrutiva do Sono no Adulto. São Paulo: Estação Brasil, 2013.

9. Araújo MFM, Freitas RWJF, Lima ACS, Pereira DCR, Zanetti ML, Damasceno MMC. Indicadores de saúde associados com a má qualidade do sono de universitários. Rev Esc Enferm USP. 2014; 48(6):108592.

10. Barnes M, Goldsworthy UR, Cary BA, Hill CJ. A diet and exercise program to improve clinical outcomes in patients with obstructive sleep apnea - a feasibility study. J Clin Sleep Med. 2009; 5(5):409-15.

11. Kline CE, Crowley EP, Ewing GB, JB Burch, Blair SN, Durstine JL, Davis JM, Youngstedt SD. The effect of exercise training on obstructive sleep apnea and sleep quality: a randomized controlled trial. Sleep. 2011; 34(12):1631-40.

12. Kline CE, Ewing GB, Burch JB, Blair SN, Durstine JL, Davis JM et al. Exercise training improves selected aspects of daytime functioning in adults with obstructive sleep apnea. J Clin Sleep Med. 2012; 8(4):357-65.

13. Passos GS, Poyares DLR, Santana MG, Tufik S, Mello MT. Is exercise an alternative treatment for chronic insomnia? Clinics. 2012;67(6):653-9

14. Pereira ECA, Schmitt ACB, Cardoso MRA, Pereira WMP, Lorenzi-Filho G, Blumel JE et al. Prevalência da sonolência diurna excessiva e fatores associados em mulheres de 35 a 49 anos de idade do "Projeto de Saúde de Pindamonhangaba" (PROSAPIN). Rev Assoc Med Bras. 2012; 58(4):447-52.

15. Ashrafinia F, Mirmohammadali M, Rajabi $H$, Kazemnejad A, Sadeghniiathaghighi K, Amelvalizadeh $\mathrm{M}$, et al. The effects of Pilates exercise on sleep quality in postpartum women J Bodyw Mov Ther. 2014; 18(2):190-9.

16. Monteiro N T, Neri A L, Ceolim M F. Sintomas de insônia, cochilos diurnos e atividades físicas de lazer em idosos: estudo FIBRA Campinas. Rev Esc Enferm USP. 2014; 48(2):242-9. 


\section{CONFLITO DE INTERESSES}

Os autores declaram não haver conflitos de interesse.

\section{AUTOR PARA CORRESPONDÊNCIA}

Silke Anna Theresa Weber silke@fmb.unesp.br

Submetido em 11/09/2017

Aceito em 30/11/2017 